\title{
ChromContact: A web tool for analyzing spatial contact of chromosomes from $\mathrm{Hi}-\mathrm{C}$ data
}

Tetsuya Sato ${ }^{1,2}$ and Mikita Suyama ${ }^{1,2^{*}}$

\begin{abstract}
Background: $\mathrm{Hi}-\mathrm{C}$ analysis has revealed the three-dimensional architecture of chromosomes in the nucleus. Although $\mathrm{Hi}-\mathrm{C}$ data contains valuable information on long-range interactions of chromosomes, the data is not yet widely utilized by molecular biologists because of the quantity of data.

Results: We developed a web tool, ChromContact, to utilize the information obtained by Hi-C. The web tool is designed to be simple and easy to use. By specifying a locus of interest, ChromContact calculates contact profiles and generates links to the UCSC Genome Browser, enabling users to visually examine the contact information with various annotations.

Conclusion: ChromContact provides wide-range of molecular biologists with a user-friendly means to access high-resolution Hi-C data. One of the possible applications of ChromContact is investigating novel long-range promoter-enhancer interactions. This facilitates the functional interpretation of statistically significant markers identified by GWAS or ChIP-seq peaks that are located far from any annotated genes. ChromContact is freely accessible at http://bioinfo.sls.kyushu-u.ac.jp/chromcontact/.
\end{abstract}

Keywords: Hi-C, Long-range interaction, Enhancer

\section{Background}

$\mathrm{Hi}-\mathrm{C}$ can detect genome-wide three-dimensional (3D) chromatin interactions by cross-linking spatially proximal genomic fragments followed by high-throughput pairedend DNA sequencing [1]. Hi-C was successfully applied to unveil genome-wide long-range interactions [2] such as the relationship between distant enhancers and promoters. Recently reported Hi-C experiments identified a huge amount of spatial contact between genomic fragments, which contain known distant enhancers and their target genes, at 5$10-\mathrm{kb}$ resolution [3]. More recent experiments achieved an even higher resolution [4].

Although the number of cell types with $\mathrm{Hi}-\mathrm{C}$ data is limited, it is shown that megabase-sized chromatin domains are not significantly different between distinct cell types and even between species [2]; this indicates

\footnotetext{
* Correspondence: mikita@bioreg.kyushu-u.ac.jp
${ }^{1}$ Medical Institute of Bioregulation, Kyushu University, Fukuoka 812-8582,

* Correspondence: mikita@bioreg.kyushu-u.ac.jp
${ }^{1}$ Medical Institute of Bioregulation, Kyushu University, Fukuoka 812-8582, Japan

${ }^{2}$ AMED-CREST, Japan Agency for Medical Research and Development, Fukuoka 812-8582, Japan
}

(C) 2015 Sato and Suyama. Open Access This article is distributed under the terms of the Creative Commons Attribution 4.0 International License (http://creativecommons.org/licenses/by/4.0/), which permits unrestricted use, distribution, and reproduction in any medium, provided you give appropriate credit to the original author(s) and the source, provide a link to the Creative Commons license, and indicate if changes were made. The Creative Commons Public Domain Dedication waiver (http://creativecommons.org/publicdomain/zero/1.0/) applies to the data made available in this article, unless otherwise stated. by $\mathrm{Hi}-\mathrm{C}$ analysis can be transferred to other tissues or species to infer spatial contact in chromosomes from other samples. Moreover, the number of cell types with $\mathrm{Hi}-\mathrm{C}$ data has recently expanded [4], and this will increase in the future.

Despite the fact that Hi-C data contains valuable information on 3D interactions within and between chromosomes, the data is not yet widely utilized by molecular biologists, mainly because of the quantity of data and complicated procedures used to calculate normalized contact matrices. To overcome this barrier, we developed a user-friendly web tool, ChromContact, for analysis of Hi-C data. Compared with currently available web-based analysis tools, such as 3DGD [5], the WashU Epigenome Browser [6], Juicebox [4], and Virtual 4C (http://promoter.bx.psu.edu/hi-c/virtual4c.php), ChromContact provides additional features including the following: (i) simple input specification; (ii) contact profile visualization in the UCSC Genome Browser via Track Hubs [7], which is capable of displaying several Hi-C 


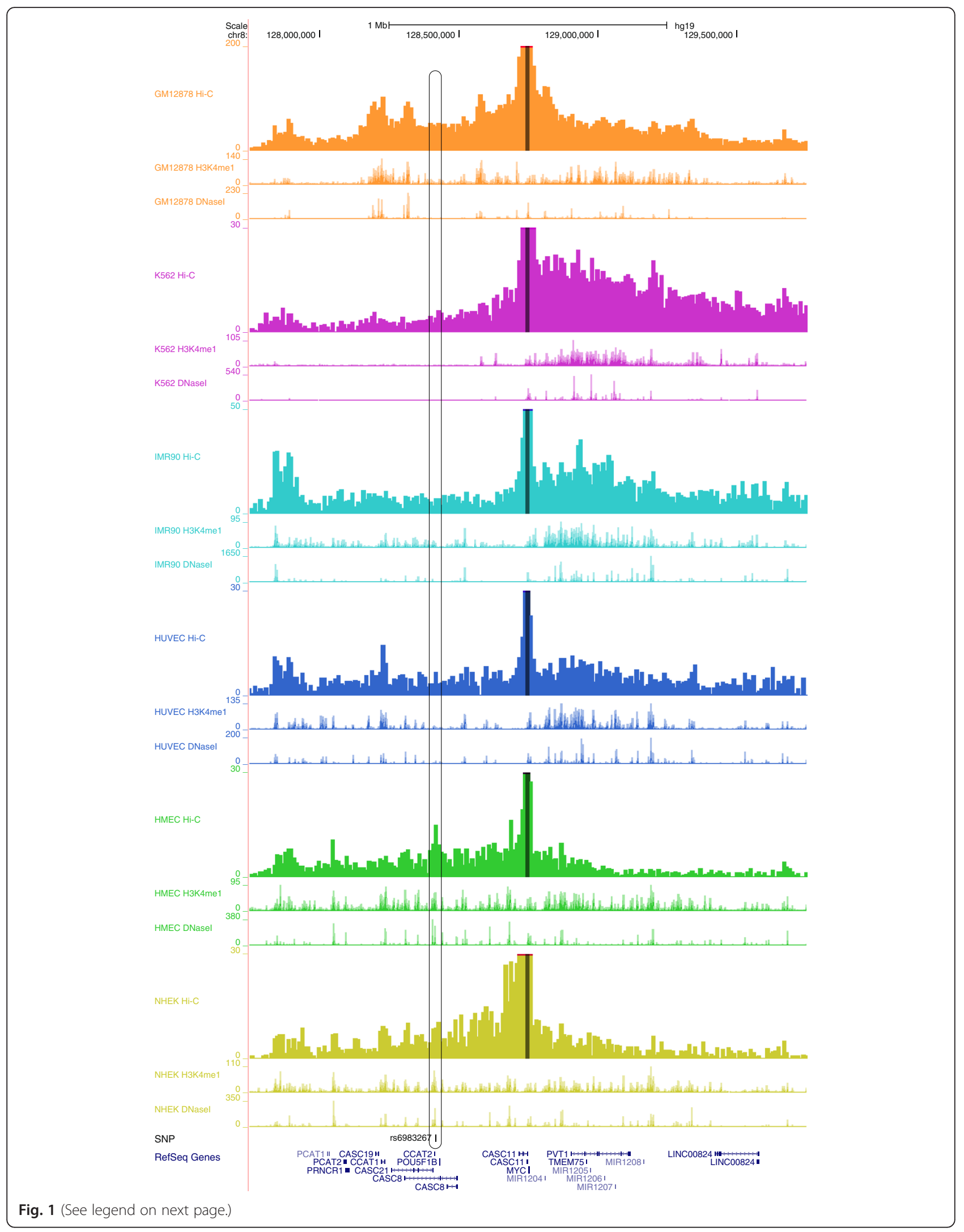


(See figure on previous page.)

Fig. 1 An example of a known long-range enhancer-promoter interaction in the human MYC locus. A 2-Mb genomic interval around the human MYC locus (genome assembly hg19) is shown in the UCSC Genome Browser. There are three tracks (from the top): (i) contact profile at 10-kb resolution obtained from Hi-C data; (ii) profile for H3K4me1; and (iii) profile for DNase I hypersensitivity sites, for each of the six cell types (GM12878, K562, IMR90, HUVEC, HMEC, and NHEK). Different colors are used for each of the six cell types. The anchor, which contains the transcription start site of MYC, is indicated by dark-colored highlights in the contact profiles. The colorectal cancer associated SNP, rs6983267, and gene annotations are shown at the bottom. The position of the SNP and the corresponding peaks in the profiles are indicated by a rounded rectangle

data records for comparison with various annotations; (iii) analysis of multiple loci of interest at a single time; and (iv) output in text format for further downstream analyses.

\section{Implementation}

We used high-resolution $\mathrm{Hi}-\mathrm{C}$ data [4] deposited in the Gene Expression Omnibus (GEO) [8] with the accession number GSE63525. We downloaded contact matrices and normalization vector files registered under this accession to use in the ChromContact server. The current version of ChromContact covers six human cell types (GM12878, K562, IMR90, HUVEC, HMEC and NHEK) at three resolutions: $5 \mathrm{~kb}, 10 \mathrm{~kb}$, and $25 \mathrm{~kb}$. We used this data because it is the highest resolution $\mathrm{Hi}-\mathrm{C}$ data available so far. We will update the data as new high resolution data is available. Contact matrices were normalized beforehand by applying normalization vector files. Because our main interest was to examine detailed structural interactions, such as enhancer-promoter interactions within a chromosome, we focused only on intrachromosomal interactions. Pre-calculated normalized contact matrices for a single sample occupy approximately 5.7 Gbyte (25-kb resolution) of server disk space. To extract contact information for a region of interest in a time- and memory-efficient manner, a random access function was implemented in our backend scripts.

Although Hi-C data is usually represented as a twodimensional contact matrix, this makes it difficult to visually compare multiple $\mathrm{Hi}-\mathrm{C}$ data with each other. Therefore, in ChromContact, we adopted 4C-like representation by specifying a user defined anchor coordinate to obtain a contact profile for the anchor, instead of directly using the two-dimensional contact matrix.

For the input, ChromContact uses a gene symbol, single nucleotide polymorphism (SNP) ID from a genome-wide association study (GWAS) Catalog [9], or a genomic coordinate. To analyze multiple loci, users may specify the input genomic coordinates in BED format, which is widely used in high-throughput genome analyses. In the case of gene symbol as an input, ChromContact automatically convert it to the coordinates of transcription start sites for the gene. The input coordinate is first converted to the corresponding anchor position, calculated based on the predefined resolution (5-, 10-, or 25-kb). A contact profile for the anchor (representing spatial proximity to the anchor for all chromosomal regions in a given resolution along the entire chromosome) is then generated from the normalized contact matrix.

In the option settings, the users may specify cell types and the resolutions to generate contact profiles. Although the highest resolution prepared in ChromContact is $5-\mathrm{kb}$ for the contact profile, it often results in sparse data for the contact profile with many fluctuations, which makes it difficult to distinguish them from small but real peaks. For this reason, we adopted $10-\mathrm{kb}$ resolution as a default parameter to obtain smoother contact profiles.

For the output, a link to the UCSC Genome Browser [10] containing the retrieved contact profile as the Track Hubs data [7] is generated to provide the user with a visual and interactive interface for detailed comparisons to up-to-date annotations stored in the UCSC Genome Browser. Since our main interest is to identify long-range enhancer-promoter interaction, ChromContact generates the Track Hubs data that contains not only the contact profile but also the profiles of H3K4me1 and DNaseI hypersensitivity sites by default. After opening the UCSC Genome Browser from the ChromContact output, the user can add other annotation tracks either by selecting the desired data stored in the UCSC Genome Browser or by uploading the user's own data as a custom track. The user-friendly functionalities of the UCSC Genome Browser facilitate easy operation, for example zooming and scrolling, of the browser display.

For another output format, ChromContact also generates a list of regions with normalized contact counts in a text format, which facilitates further text-based downstream analyses; for example, sorting the results by contact counts using conventional spreadsheet applications.

\section{Results and discussion}

Here we demonstrate an example using the known longrange enhancer-promoter interaction in the human $M Y C$ locus (genome assembly hg19) (Fig. 1). SNP rs6983267, located 335-kb upstream of $M Y C$, is strongly associated with colorectal cancer through spatial contact between SNP positioned in the $M Y C$ enhancer and promoter [11, 12]. By specifying the genomic coordinates of the $M Y C$ promoter as the anchor position and selecting all the six cell types for $\mathrm{Hi}-\mathrm{C}$ data sets, ChromContact calculated the contact profiles for each of them. As shown in the UCSC Genome Browser output (Fig. 1), there is a prominent peak at the 


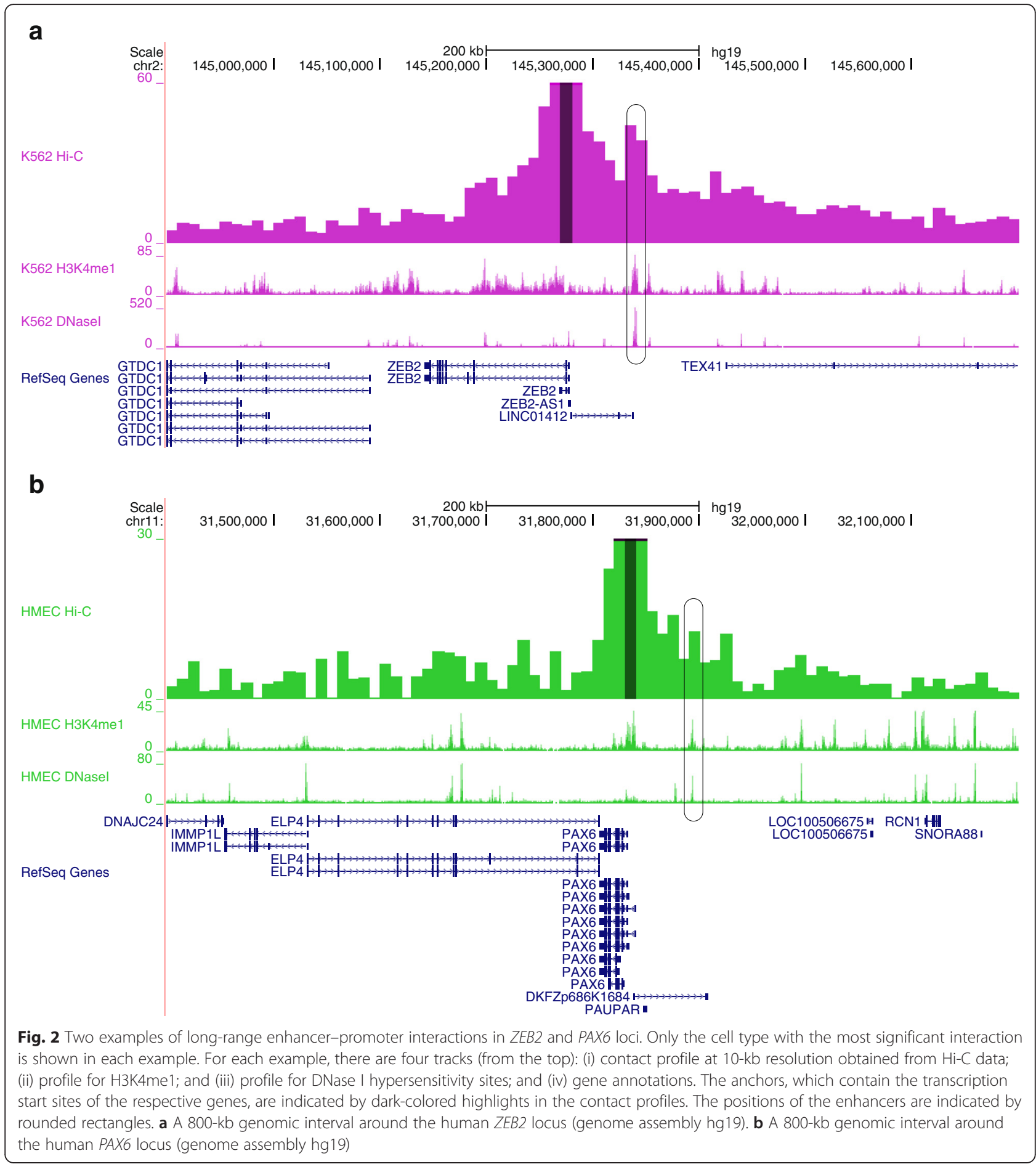

rs6983267 in the contact profile for HMEC cells (approximately chr8:128,413,000), indicating that the genomic fragment containing SNP rs6983267 can interact with the anchor (corresponding to the $M Y C$ promoter region) through chromosomal loop formation in HMEC cells. By comparing with ENCODE epigenomic data [13], H3K4me1, a histone mark for an enhancer, was observed in the same chromosomal position in HMEC cells, suggesting that the site may act as an enhancer. A DNase I hypersensitive site was also observed at this position. This indicates that the known distal enhancer-promoter interaction can be identified by incorporating currently available $\mathrm{Hi}-\mathrm{C}$ and other epigenomic data. At the same genomic position, NHEK also has a 
peak in the profiles of $\mathrm{Hi}-\mathrm{C}$ contact, $\mathrm{H} 3 \mathrm{~K} 4 \mathrm{me}$, and DNase I hypersensitivity. The cell-type specificity of the $\mathrm{Hi}-\mathrm{C}$ peak at this position well explain their association with colorectal cancer, i.e., both HMEC and NHEK belong to epithelial cells, from which most of colorectal cancer is developed. The same peak was not observed neither in hematopoietic cells (GM12878 and K562) and the other cell types (IMR90 and HUVEC). In the contact profiles, there are other peaks that correspond to H3K4me1 mark and DNase I hypersensitivity site in the matched cell types. These peaks may contain candidates for other $M Y C$ enhancers with cell-type specificity. As shown in this example, contact profiles are not always very similar between different cell types in rather high resolution such as $10-\mathrm{kb}$, but differ according to groups of cell types. This indicates that it is important to analyze $\mathrm{Hi}-\mathrm{C}$ data from a cell type that is close to the one that the user is interested in, or to analyze $\mathrm{Hi}-\mathrm{C}$ data from as many cell types as possible.

Next, we present two examples, for which long-range enhancer-promoter interactions had not been supported so far by $3 \mathrm{C}, 4 \mathrm{C}$, or Hi-C experiments (Fig. 2). The first example is a long-range enhancer-promoter interaction found in ZEB2 locus (Fig. 2a). It is reported that one of the enhancers for $Z E B 2$ conserved among human, mouse, and rat, and the enhancer is located about $65 \mathrm{~kb}$ upstream of the transcription start site of ZEB2 in human [14]. In the contact profile, there is a peak that corresponds to H3K4me1 mark and DNase I hypersensitivity site, indicating that the enhancer region is spatially close to the promoter of ZEB2 (Fig. 2a). The second example is a long-range enhancer-promoter interaction found in PAX6 locus (Fig. 2b). There is a peak in the contact profile, which also corresponds to H3K4me1 mark and DNase I hypersensitivity site. For this region, enhancers for PAX6 are identified in the corresponding region in mouse [15]. These data support the idea that the peak region contains enhancers for PAX6 also in human, and that they are spatially proximal to the promoter of PAX6 in HMEC cells.

\section{Conclusions}

Increasing examples show that mutations not only in coding regions but also in regulatory regions are responsible for genetic diseases [16]. There are many cases in which statistically significant markers identified by GWAS or ChIP-seq peaks are located far from any annotated genes. Although there are already several known cases of longrange enhancer-promoter interactions, knowledge about these distal relationships is limited. This is mainly because identification of the relationships between distal regulatory elements and their target genes, which can be achieved via 3D architecture contact, has not been an easy task and requires laborious experiments. The recently devised $\mathrm{Hi}-\mathrm{C}$ method, which reveals spatial interactions on a genomewide scale, combined with rapid and continuous improvements of the method will dramatically change the means to analyze long-range interactions. ChromContact provides molecular biologists with a user-friendly means to access to high-resolution $\mathrm{Hi}-\mathrm{C}$ data and facilitate analysis of long-range chromosomal interactions.

\section{Availability and requirements}

Project name: ChromContact.

Project home page: http://bioinfo.sls.kyushu-u.ac.jp/ chromcontact/.

Operating system(s): Platform independent (web-based). Programming language: Perl, php.

Other requirements: None.

License: GPL-3.0.

Any restrictions to use by non-academics: No restrictions.

\section{Ethics statement}

Ethics statement is not applicable to our study as this study only uses publicly available data.

\section{Abbreviations \\ 3D: Three-dimensional; GWAS: Genome-wide association study; SNP: Single nucleotide polymorphism}

\section{Competing interests}

The authors declare that they have no competing interests.

\section{Authors' contributions}

All authors, TS and MS, designed the study, wrote the code, and prepared the manuscript. Both authors read and approved the final manuscript.

\section{Acknowledgements}

This work was supported by the Grant-in-Aid for Scientific Research from the Ministry of Education, Culture, Sports, Science and Technology of Japan (26550089 to T.S.; 21510215 and 22132005 to M.S.) and the Kyushu University Interdisciplinary

Programs in Education and Projects in Research Development (P\&P).

Received: 31 August 2015 Accepted: 8 December 2015

Published online: 15 December 2015

\section{References}

1. Lieberman-Aiden E, van Berkum NL, Williams L, Imakaev M, Ragoczy T, Telling A, et al. Comprehensive mapping of long-range interactions reveals folding principles of the human genome. Science. 2009;326:289-93.

2. Dixon JR, Selvaraj S, Yue F, Kim A, Li Y, Shen Y, et al. Topological domains in mammalian genomes identified by analysis of chromatin interactions. Nature. 2012:485:376-80

3. Jin F, Li Y, Dixon JR, Selvaraj S, Ye Z, Lee AY, et al. A high-resolution map of the three-dimensional chromatin interactome in human cells. Nature. 2013; 503:290-4.

4. Rao SS, Huntley MH, Durand NC, Stamenova EK, Bochkov ID, Robinson JT, et al. A 3D map of the human genome at kilobase resolution reveals principles of chromatin looping. Cell. 2014;159:1665-80.

5. Li C, Dong X, Fan H, Wang C, Ding G, Li Y. The 3DGD: A database of genome 3D structure. Bioinformatics. 2014;30:1640-2.

6. Zhou X, Lowdon RF, Li D, Lawson HA, Madden PA, Costello JF, et al. Exploring long-range genome interactions using the WashU Epigenome Browser. Nat Methods. 2013;10:375-6.

7. Raney BJ, Dreszer TR, Barber GP, Clawson H, Fujita PA, Wang T, et al. Track data hubs enable visualization of user-defined genome-wide annotations on the UCSC Genome Browser. Bioinformatics. 2014;30:1003-5. 
8. Barrett T, Wilhite SE, Ledoux P, Evangelista C, Kim IF, Tomashevsky M, et al. NCBI GEO: Archive for functional genomics data sets - update. Nucleic Acids Res. 2013;41:D991-5.

9. Welter D, MacArthur J, Morales J, Burdett T, Hall P, Junkins H, et al, The NHGRI GWAS Catalog, a curated resource of SNP-trait associations. Nucleic Acids Res. 2014;42:D1001-6.

10. Rosenbloom KR, Armstrong J, Barber GP, Casper J, Clawson H, Diekhans M, et al. The UCSC Genome Browser database: 2015 update. Nucleic Acids Res. 2015;43:D670-81.

11. Pomerantz MM, Ahmadiyeh N, Jia L, Herman P, Verzi MP, Doddapaneni $H_{\text {, }}$ et al. The 8q24 cancer risk variant rs6983267 shows long-range interaction with MYC in colorectal cancer. Nat Genet. 2009;41:882-4.

12. Tuupanen S, Turunen M, Lehtonen R, Hallikas O, Vanharanta S, Kivioja T, et al. The common colorectal cancer predisposition SNP rs6983267 at chromosome 8q24 confers potential to enhanced Wnt signaling. Nat Genet. 2009:41:885-90.

13. ENCODE Project Consortium. An integrated encyclopedia of DNA elements in the human genome. Nature. 2012;489:57-74.

14. El-Kasti MM, Wells T, Carter DA. A novel long-range enhancer regulates postnatal expression of Zeb2: Implications for Mowat-Wilson syndrome phenotypes. Hum Mol Genet. 2012;21:5429-42.

15. Bhatia S, Monahan J, Ravi V, Gautier P, Murdoch E, Brenner S, et al. A survey of ancient conserved non-coding elements in the PAX6 locus reveals a landscape of interdigitated cis-regulatory archipelagos. Dev Biol. 2014;387: 214-28.

16. Gordon CT, Lyonnet S. Enhancer mutations and phenotype modularity. Nat Genet. 2014;46:3-4.

\section{Submit your next manuscript to BioMed Central and we will help you at every step:}

- We accept pre-submission inquiries

- Our selector tool helps you to find the most relevant journal

- We provide round the clock customer support

- Convenient online submission

- Thorough peer review

- Inclusion in PubMed and all major indexing services

- Maximum visibility for your research

Submit your manuscript at www.biomedcentral.com/submit 